\title{
The design of the RF radio signal acquisition and analysis system
}

\author{
Gaojian Zhang, Gang Yang, Lin Yang, Qingqing Zhang \\ The Communication University of China, China \\ *1198926935@qq.com
}

\section{Keywords: ARM; FPGA; DMA; Multiple frequency}

\begin{abstract}
A RF radio signal acquisition and analysis system which is based on ARM and FPGA hardware architecture is designed in the paper. The system can measure the field strength data of multiple frequency radio signals at the same time, and save the demodulation broadcasting audio in real time. In addition, with the GPS technology in the test, the signal field-strength overlays in the test area can be acquired with the further analysis of the collection data.
\end{abstract}

\section{Introduction}

With the development of space technology, RF signal acquisition and analysis has been more and more widely application in the communication areas. Analyzing the field strength coverage situation of RF radio signals in a certain area, can not only provide important reference for the building of signal transmitter, but also achieve the function of the radio signal monitoring.

The development of hardware technology is increasingly and the device which can only test the single frequency radio signal has already can't meet the needs of masses. A more powerful hardware system is needed for the analysis of RF radio signals. The system has the ability to handle multiple frequency radio signals at the same time, including the collection of signal field strength data and the preservation of the broadcast audio stream.

The system should have the high-speed sampling and the strong analyzing and processing ability for testing the data of multiple frequency signals and collecting multichannel audio at the same time. ARM processor is a high-performance and low-power hardware platform. It's good at logical operations and has the strong ability in data display and analysis. However, it's difficult for ARM to complete the real-time acquisition of a large amount of data. And FPGA has a large amounts of adder and multiplier, is good at high-speed acquisition and operation [1].

At first, completing the data acquisition work with FPGA and then sending them into ARM to analysis and preservation. The collecting and analyzing of multiple frequency signals can be realized in this way.

A RF radio signal acquisition and analysis system based on the ARM and FPGA architecture is designed and realized in the paper [2]. The system can deal with eight frequency signals at the same time. The demodulation broadcast audio data will be compression coding and saved. The field strength data will be saved combining with the geographic information. After the system finished testing work, the collected data can be loaded into the map software and the field strength coverage situation can be displayed on the map. The test results of RF radio signal can be displayed more intuitively.

\section{The Overall Structure Of System}

The whole system mainly consists of four parts, the GPS module, RF signal demodulation module, FPGA module and ARM processor module. The system structure diagram as shown in the figure below: 


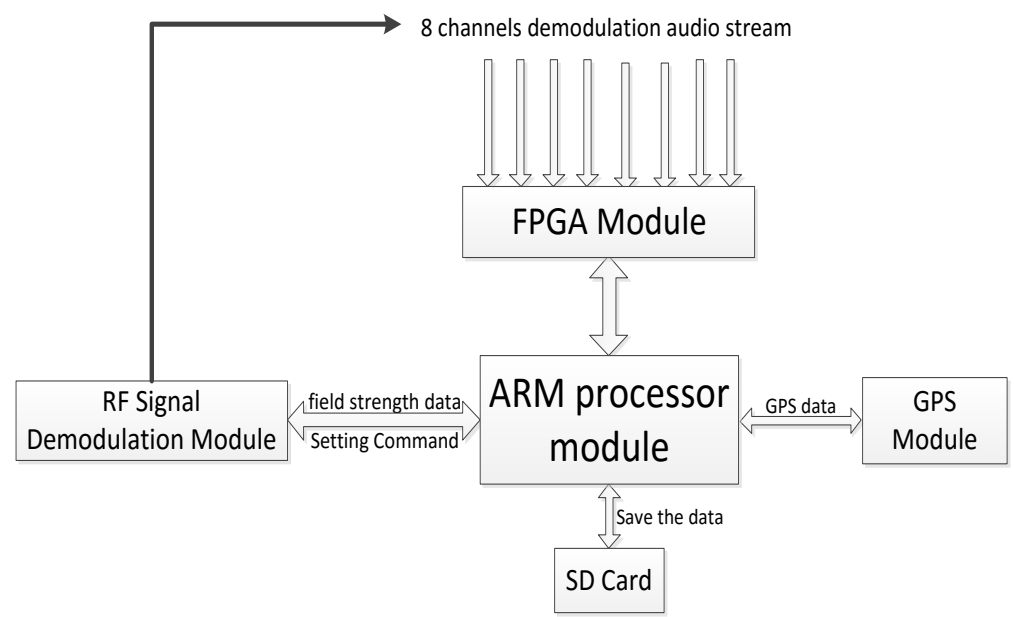

Figure 1. The overall structure of system

While the system is powered on, the GPS signal module is in the working state and begins to collect the location information in real time. After the antenna receiving the RF radio signal, the RF signal demodulation module starts to complete the radio field strength data acquisition and the audio signal demodulation. And then the FPGA module collects the demodulation audio stream of eight channels, and the ARM processor receives the field strength data of eight frequency signals at the same time. Next, the FPGA module will transmit the audio data to the ARM processor through the hardware interface. ARM receives the data and completes the audio compression coding and saving work. At last, ARM needs to save the received field strength data with the geographic information together. All data can be saved into the external SD card.

\section{The Hardware Modules}

The RF signal demodulation module. The main function of the module is to analyze the RF radio signal, and get the relevant data of field strength and broadcast audio. The core chip of the module in the system is the Si4721 chip which is produced by Silicon Labs. This is a single chip of FM radio transceiver chip, provide complete FM transceiver functions, can easily transplanted to a portable device [3].

The FM module which is based on Si4721 chip can complete the work for radio signal demodulation and quality testing. The hardware circuit diagram of the module is shown below:

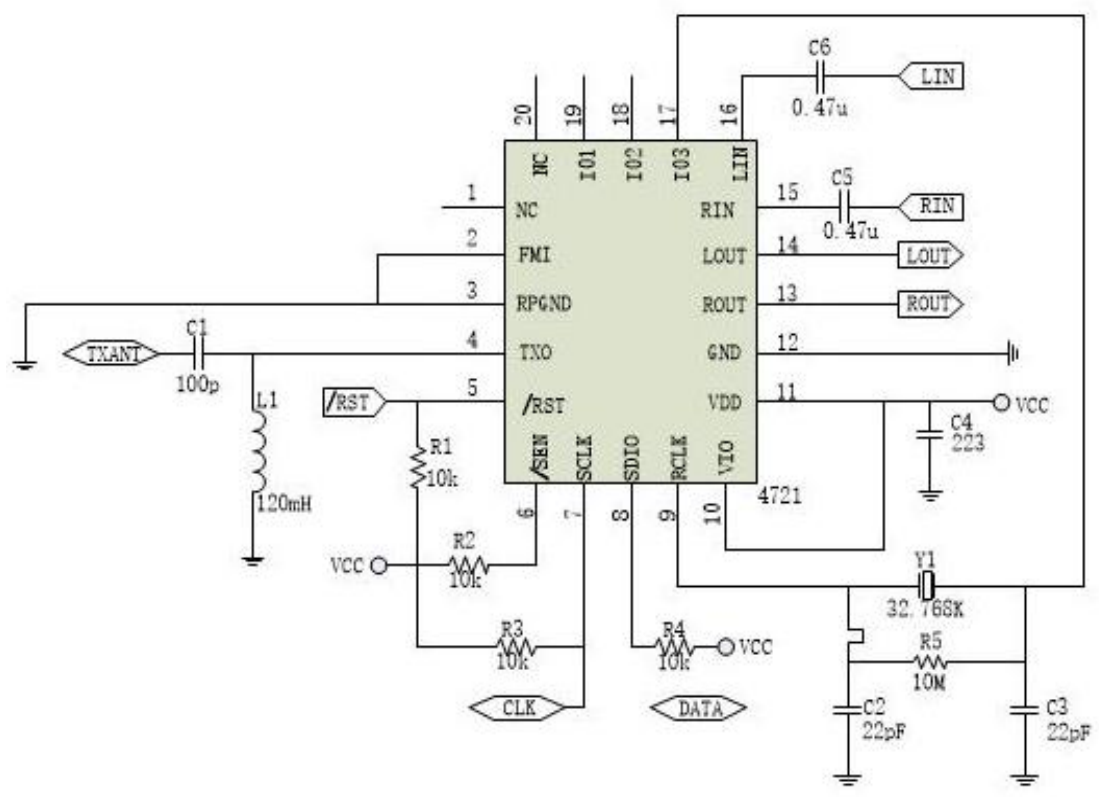

Figure 2. The hardware circuit diagram of demodulation module 
The control mode of the module is 2 - wire mode (I2C mode), connected to the ARM processor through the $\mathrm{I} 2 \mathrm{C}$ interface, receiving the instruction from ARM and completing relevant work.

The GPS Module. The test for RF signal at most time is not concerned about the signal strength at a certain point, but in the whole test area. Knowing the signal strength of a frequency signal in a certain area can bring help for subsequent work. So it's necessary to record the location information at the time of collecting and analyzing the RF radio signals. It is convenient to draw field strength coverage map of the RF radio signal.

The system collects geographic information using the GPS navigation module with the RS232 serial port. The module adopts high sensitivity navigation chip SIRF3 and the default communication rate is 9600 Baud. The module is automatically working while powering up, and data from the serial port is standard NMEA0183 format. A series of information such as longitude, latitude, altitude, and the instantaneous velocity of equipment are all in the data.

The FPGA Module. The main function of FPGA module is to complete the real-time acquisition of eight-channel audio data stream because ARM processor is not good at this kind of work. However, it's important to send the audio data to the ARM processor after the acquisition. The communication between ARM and FPGA is a typically asynchronous communication. It's a difficult technology for the high-speed asynchronous communication with a large amount of data. This is a very important part of the whole system design.

The FPGA module is used DE2-115 development board from Terasic Company, built-in Altera CycloneIV 4CE115 FPGA chip [4]. There's expansion interface with secondary protection circuit to implement the communication between with ARM.

The ARM Processor Module. The ARM processor is the core of the whole system and is the most important part of it. ARM is not only dealing with and saving all the data, but also controlling and managing the working of all modules. The system adopts i.MX6 processor produced by Freescale Semiconductor Company as the ARM processor.

The i.MX6 series processor is based on Cortex-A9 processor architecture, and contains the single-core, dual-core and four-core hardware products. It's very suitable for the design because i.MX6 has high performance multimedia processing ability and powerful compatibility for pins and software [5].

The Hardware Interface. Without the single hardware module, the hardware interface for connecting the various modules in the design is also an important part. The ARM processor and radio signal demodulation module are connected by I2C interface, and with GPS module are connected by RS232 serial port. The two kinds of interface can be directly opened and called.

However, it's needed to choose the appropriate interface for the connection between the ARM and FPGA. A bus interface called EIM (External Interface Module) in the i.MX6 is used in the system. The EIM bus interface is exterior interface module bus interface, with 28 bits of external address bus and the maximum memory to $256 \mathrm{MB}$. The most important reason for choose this interface is that the bus uses the AXI bus protocol and support the DMA transmission technology. The DMA technology is the key to realize high speed asynchronous communication between ARM and FPGA [6].

\section{The Software Design}

The DMA Program. Realizing the asynchronous high-speed transmission of large amounts of data between ARM and FPGA is a technical difficulty in the whole system. Eventually the DMA transfer technology is adopted to complete this function.

The DMA is short of Direct Memory Access. It's a kind of high speed data transmission operation technology, and allowing the data are read and written directly between the external devices and memory [7]. The process is not by the CPU and needn't the intervention of CPU. The whole data transmission operation is in the control of the DMA controller, and the efficiency can be greatly improved in this way.

The first operation is to load the correct DMA driver on the ARM if the system wants to use the DMA technique. The DMA driver program is a character device driver program essentially. In the Linux system, the DMA transfers can be realized according to the process in the figure 3. 


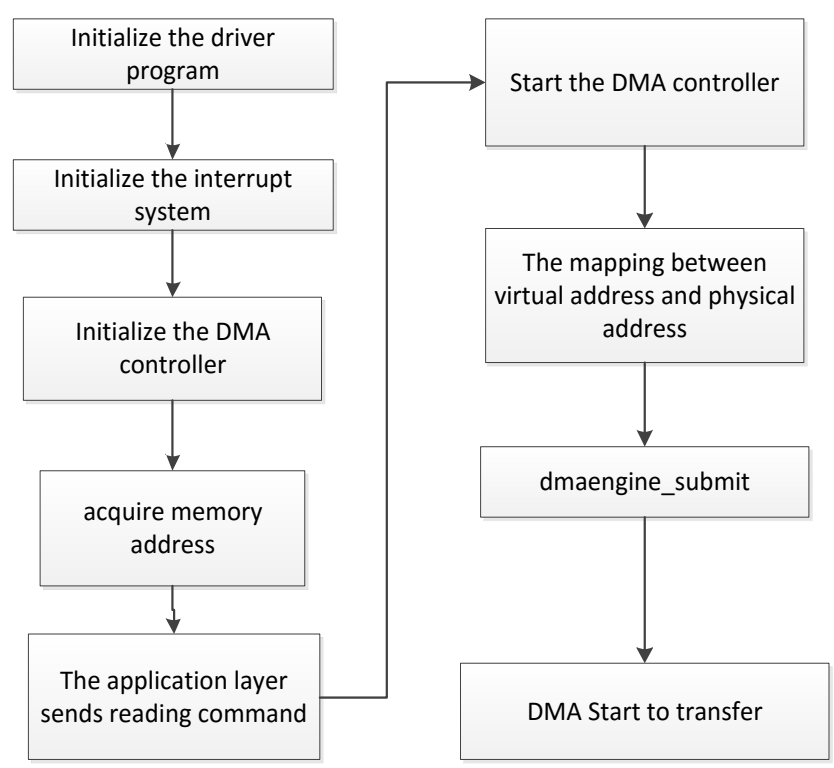

Figure 3. The implementation process of DMA

At first, the DMA driver program starts the initialization and completes the setting of related registers and storage area. Then initializing the interrupt system and configuring the trigger mode of the external interrupt. Finally, initializing the DMA controller and setting the DMA channel number and the source address. Applying for the DMA transmission memory and obtaining the memory address as the target address of DMA transfers.

The initialization process is completed at this time and need to wait for the read data command from the application layer, then making sure the FPGA is ready for data. The DMA transfers can be implemented. It is important to note that the CPU transmission is working by virtual address, but the DMA transfer is by physical address. It's necessary to complete the mapping from virtual address and physical address before starting the DMA and then begin the DMA transfer. At the end of the DMA transfer, calling a function to the CPU to send interrupt, and waiting for the data to be taken to complete the DMA transfer operation [8].

The ARM processor function program. The software part of ARM processor mainly includes two aspects of function.

Controlling the other function modules. The function modules which are connected to the ARM, including RF signal demodulation module, GPS module and FPGA module, needed to be controlled by ARM.

The signal demodulation module connects to the ARM by the I2C interface and needs to receive the instructions from ARM to complete the relevant work. The ARM software program should according to the electrical characteristics of Si4721 chip, send the corresponding data and analyzing of the returned data. Finally get the field strength data and demodulation audio stream.

The GPS module is working automatically with electricity and sends the data through the serial port. The mainly function of the software program is to open the serial port and get the location information we needed according to the NMEA0183 format.

For the FPGA section, it's necessary to add an application program after loading the DMA driver successfully. The function of the program is to guarantee the data extraction and interrupt control.

Processing and saving the collected data. The audio stream sending by FPGA needs to be compressed coding and saved. All the work is done by the program of ARM. At the same time, the data from the demodulation module and GPS module are also need to be saved by ARM program.

\section{The Results and Conclusion}

The figure 4 shows the display effect in the web, with the collected data combining with the Baidu map API. There are different colors of cover points to represent the signals with different intensity. Each position has different field strength data for different frequency points. The data can be 
displayed in the information window of web page. The signal intensity in the whole area can be observed visually in this way.

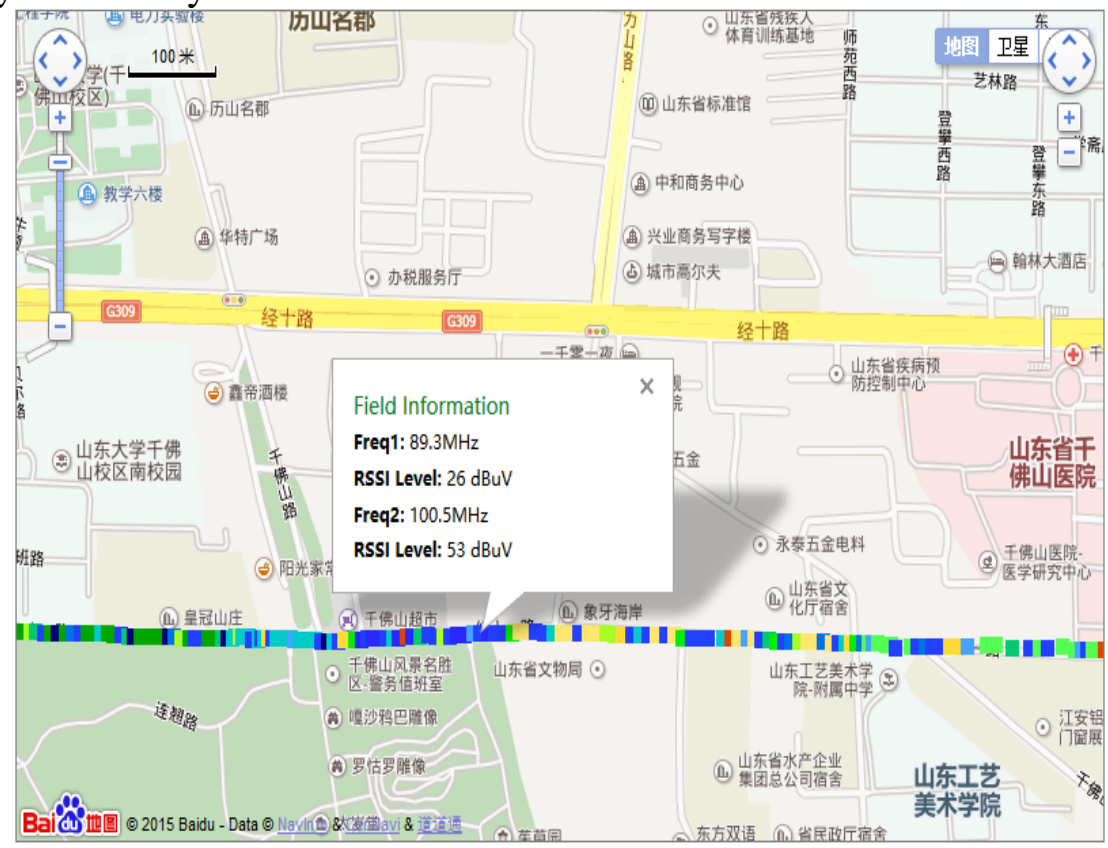

Figure 4. The display effect of collected data in the map

In fact, the ARM and FPGA hardware scheme in the system is not only can be applied to radio signal acquisition and analysis, but also can be applied to other RF signal analysis system. The framework can realize the RF signal processing for the terrestrial digital TV and $3 \mathrm{G}$ mobile signal as long as making the change to the front demodulation module. It provides a good hardware options to the RF signal processing.

\section{References}

[1] Zhang Haifeng, Zhao Jing, "The design of RF data acquisition system based on STM32 and FPGA," 2011.

[2] Li Nianqiang, Wei Changzhi, Pan Jianjun and Zhang Yu, Data Acquisition System and System Design, 1st ed., China Machine Press: Beijing, 2009.

[3] “Si47XX Programming Guide,” Rev. 0.7 6/11 (Silicon Labs, Inc,2010).

[4] “DE2-115 User Manual,” Rev 1.0 (Terasic, Inc,2013).

[5] “i.MX 6Dual/6Quad Multimedia Applications Processor Reference Manual,” Rev. C (Freescale, Inc,2012).

[6] “ARM Limited lnc. AMBA AXI Protocol specification,” REV1.0 [EB/OL]. 1999-05-13.

[7] LiBo, LiuDatong, and PengYu, "A High Speed DMA Transaction Method for PCI Devices," Journal of Electronic Measurement and Instrument, vol.22, pp. 705-710, Aug. 2008.

[8] WANG Xiaoping, WANG Wangang, CAI Chuan, "The DMA data acquisition program design between FPGA and ARM in the Embedded Linux," Journal of Southwest Normal University, 2014 